\title{
Narrow Fano resonances in Si nanocylinder metasurfaces: Refractive index sensing
}

Cite as: J. Appl. Phys. 125, 183103 (2019); https://doi.org/10.1063/1.5094417

Submitted: 01 March 2019 . Accepted: 22 April 2019 . Published Online: 13 May 2019

Diego R. Abujetas (D), Juan J. Sáenz (D), and José A. Sánchez-Gil (D)

\section{COLLECTIONS}

Paper published as part of the special topic on Dielectric Nanoresonators and Metamaterials Note: This paper is part of the Special Topic on Dielectric Nanoresonators and Metamaterials.
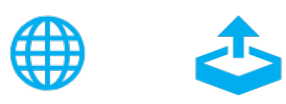

\section{ARTICLES YOU MAY BE INTERESTED IN}

Lattice resonances in dielectric metasurfaces

Journal of Applied Physics 125, 213105 (2019); https://doi.org/10.1063/1.5094122

Fano interferences of electromagnetic modes in dielectric nanoblock dimers

Journal of Applied Physics 125, 063103 (2019); https://doi.org/10.1063/1.5063403

Dielectric nanoresonators and metamaterials

Journal of Applied Physics 126, 150401 (2019); https://doi.org/10.1063/1.5129100

\section{Lock-in Amplifiers up to $600 \mathrm{MHz}$}
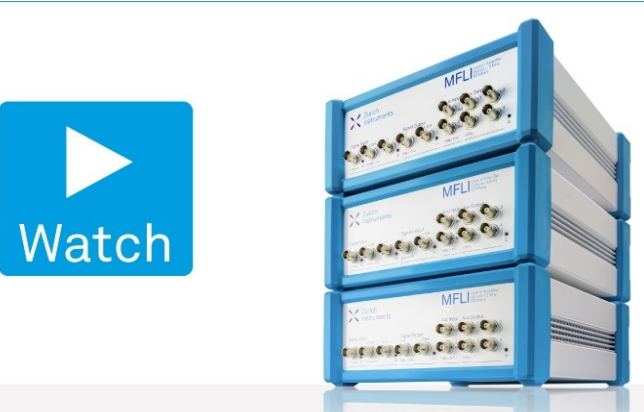


\title{
Narrow Fano resonances in Si nanocylinder metasurfaces: Refractive index sensing
}

\author{
Cite as: J. Appl. Phys. 125, 183103 (2019); doi: 10.1063/1.5094417 \\ Submitted: 1 March 2019 . Accepted: 22 April 2019. \\ Published Online: 13 May 2019
}

Diego R. Abujetas, ${ }^{1,2, a)}$ (iD Juan J. Sáenz, 2,3,b) (iD and José A. Sánchez-Gil (,c) (D)

\begin{abstract}
AFFILIATIONS
${ }^{1}$ Instituto de Estructura de la Materia (IEM-CSIC), Consejo Superior de Investigaciones Científicas, Serrano 121, 28006 Madrid, Spain

${ }^{2}$ Donostia International Physics Center DIPC, Paseo Manuel de Lardizabal 4, 20018 Donostia-San Sebastián, Spain

3IKERBASQUE, Basque Foundation for Science, 48013 Bilbao, Basque Country, Spain
\end{abstract}

Note: This paper is part of the Special Topic on Dielectric Nanoresonators and Metamaterials.

a)Electronic mail: diego.romero@iem.cfmac.csic.es

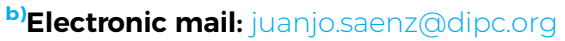

${ }^{c)}$ Electronic mail: j.sanchez@csic.es

\begin{abstract}
All dielectric metasurfaces are attracting great attention for their potential to control light reflection and transmission at the nanoscale. Here, we make use of a coupled electric/magnetic dipole formulation for a periodic array of high-refractive-index cylinders to analytically explore the emergence of Fano resonances for both $s$ - and p-polarized waves. Strong Fano resonances with large Q-factors are found in Si nanocylinder metasurfaces as a result of the lattice-induced interplay between electric and magnetic dipole resonances, where the narrow resonances are connected to bound states in the continuum supported by the lattice. The impact on the refractive index of the surrounding medium is analyzed, revealing that such simple all dielectric metasurfaces are suitable for refractive-index sensing devices with figures of merit of two orders of magnitude.
\end{abstract}

Published under license by AIP Publishing. https://doi.org/10.1063/1.5094417

\section{INTRODUCTION}

Narrow resonances in optics and electromagnetism are extremely appealing both from fundamental standpoints (high Q-factors, longlived states, and energy concentration) and from the obvious implications that the resulting phenomenology may have in photonics applications (e.g., sensing, lasing, nonlinear effects, enhanced spectroscopies, etc.). Much interest has been devoted in the particular case of Fano resonances: asymmetric, typically narrow line shapes that arise as the interference of a narrow dark resonance with a broad bright one, ${ }^{1-3}$ the latter being a continuum in the context of atom physics as introduced by Fano. Among the variety of configurations that exhibit Fano resonances, ${ }^{4-9}$ subwavelength arrays of scatterers called metasurfaces are especially interesting for their enhanced collective response, favoring experimental feasibility and applications. ${ }^{2,3,10-13}$

In this regard, high-refractive index (HRI) nanophotonic structures supporting strong magnetic dipole resonances in the optical domain ${ }^{14-17}$ have been proposed as a nearly lossless alternative to plasmonic nanostructures for building blocks of metamaterials ${ }^{18,19}$ and especially of metasurfaces with a variety of effective optical properties: such as negative index ${ }^{20}$ and zero index, ${ }^{21}$ broadband polarization conversion, ${ }^{21}$ perfect reflectors or absorbers, ${ }^{22-26}$ Brewster and Kerker effects, ${ }^{27-29}$ beam deflectors ${ }^{30}$ and metalenses, ${ }^{31,32}$ Fano resonances and electromagnetically-induced transparency, ${ }^{3,33}$ and even bound states in the continuum. ${ }^{34-36}$ However, very few works have investigated the potential of HRI nanophotonic structures in refractive-index sensing, ${ }^{9,37-39}$ unlike in Plasmonics; ${ }^{11,40-44}$ in this regard, HRI nanostructured metasurfaces seem to be a suitable platform for Fano-based, refractive-index sensing that remains largely unexplored. ${ }^{4}$

Among these all-dielectric metasurfaces, one of the simplest geometries considered thus far is an array of dielectric wires. ${ }^{29,34,46-48}$ Here, we make use of a coupled electric and magnetic dipole (CEMD) formulation recently developed, ${ }^{29}$ summarized in Sec. II, to investigate the emergence of Fano resonances in the reflection and transmission spectra of an all-dielectric metasurface consisting of silicon nanocylinders. The interplay between electric/magnetic, longitudinal/transverse dipole resonances, leading to strong Fano 
resonances with high Q-factors (in turn connected to bound states in the continuum), is analyzed in detail in Sec. III. The impact of the surrounding medium on the spectral shift of specific Fano resonances is shown in Sec. IV, revealing the potential of Si-based metasurfaces for refractive-index sensing. Concluding remarks are presented in Sec. V.

\section{REFLECTION AND TRANSMISSION BY A SEMICONDUCTOR NANOCYLINDER METASURFACE THROUGH COUPLED ELECTRIC/MAGNETIC DIPOLE THEORY}

We consider an infinite array of Si cylinders, with the same radius $r$ and parallel axis along the $x$ direction, placed at $\mathbf{r}_{\mathbf{n}}=(0, n a, 0), n=0, \pm 1, \pm 2, \ldots$, where $a$ is the lattice constant, illuminated by an incident plane wave normal to the cylinder axis, as shown in Fig. 1. The relative permittivity of the cylinders is $\varepsilon=12.25$; this is a typical permittivity of lossless semiconductor in the optical domain above the gap (e.g., crystalline $\mathrm{Si}^{30,49,50}$ ). We assume that its behavior can be fully described by its electric (ED) and magnetic (MD) dipolar responses, i.e., its two lowest-order electromagnetic modes along various directions, as shown in Ref. 29.

By means of a coupled electric/magnetic dipole theory for an infinite planar array (iCEMD), developed in Ref. 29, the reflectance for each diffraction channel $R_{m}=\left|\mathfrak{r}_{\mathrm{m}}\right|^{2}$ can be derived as the energy carried out by the reflected wave divided by the incoming energy, $\mathcal{E}_{i}=k_{z} / c \mu$. The specular reflectance, $R_{0}$, for given angle of incidence $\theta$ (see Fig. 1) can be written as

$$
\begin{aligned}
R_{0}^{(p)}= & \left(\frac{k^{2}}{2 k a \cos \theta}\right)^{2} \mid \gamma^{(p)}\left(\widetilde{\alpha}_{x}^{(M)}+\widetilde{\alpha}_{z}^{(E)} \sin ^{2} \theta\right. \\
& \left.+2 k^{2} G_{b z x} \widetilde{\alpha}_{x}^{(M)} \widetilde{\alpha}_{z}^{(E)} \sin \theta\right)-\left.\widetilde{\alpha}_{y}^{(E)} \cos ^{2} \theta\right|^{2}, \\
R_{0}^{(s)}= & \left(\frac{k^{2}}{2 k a \cos \theta}\right)^{2} \mid \gamma^{(s)}\left(\widetilde{\alpha}_{x}^{(E)}+\widetilde{\alpha}_{z}^{(M)} \sin ^{2} \theta\right. \\
& \left.+2 k^{2} G_{b z x} \widetilde{\alpha}_{x}^{(E)} \widetilde{\alpha}_{z}^{(M)} \sin \theta\right)-\left.\widetilde{\alpha}_{y}^{(M)} \cos ^{2} \theta\right|^{2},
\end{aligned}
$$

where $\widetilde{\alpha}_{i}^{(j)}$ are the elements of the renormalized polarizability tensor $\widetilde{\alpha}, \gamma^{(l)}$ is the factor that couples the modes in the $x z$ plane, and $G_{b z x}$

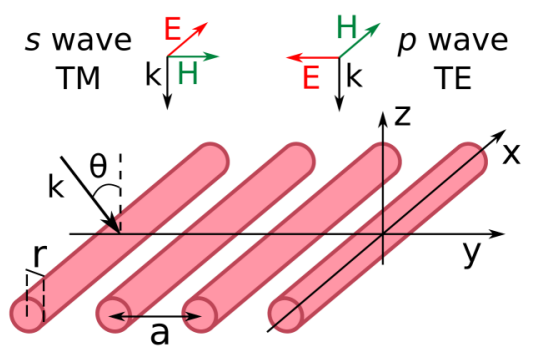

FIG. 1. Illustration of the scattering geometry of the Si-cylinder metasurface configuration.

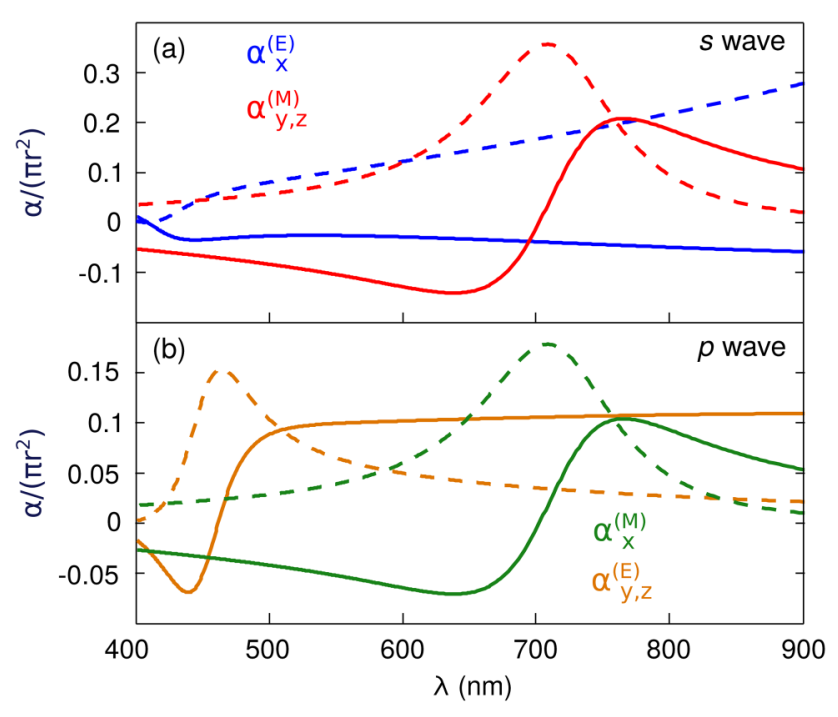

FIG. 2. Electric and magnetic polarizibilities of a Si cylinder of radius $r=75 \mathrm{~nm}$ and a relative permittivity of $\epsilon=12.25$, extracted from the first terms of Mie scattering theory (see text) for s-polarized/TM waves (top) and p-polarized/TE waves (bottom). Solid (dashed) curves correspond to real (imaginary) parts.

is the corresponding component of the lattice depolarization dyadic. ${ }^{29}$ Light polarization in the lattice geometry is referred to as $s$ and $p$ waves, corresponding to TM/TE waves for the isolated cylinders. Since there are no losses, only the reflection will be considered.

Let us study the scattering properties of an array of nonabsorbing $\mathrm{Si}$ cylinders with a ratio between the lattice parameter and the radius $a / r=4$. For the sake of completeness, we show in Fig. 2 the $r=75 \mathrm{~nm}$ cylinder polarizibilities to be used within the iCEMD, which indeed stem from the corresponding Mie coefficients. Recall that TE and TM waves are decoupled for incident field normal to the cylinder axis so that each polarization can be treated independently. ED and MD resonant modes along the cylinder axis $\left(\alpha_{x}^{(E)}, \alpha_{x}^{(M)}\right.$; longitudinal modes) stem from, respectively, $b_{o}$ and $a_{0}$ Mie coefficients, whereas $a_{1}$ and $b_{1}$ give rise to ED and MD resonant modes within the incidence plane perpendicular to the cylinder axis $\left(\alpha_{y, z}^{(E)}, \alpha_{y, z}^{(M)}\right.$; transverse modes in Fig. 2).

\section{HIGH-Q FANO RESONANCES AND ELECTROMAGNETICALLY-INDUCED TRANSPARENCY}

Let us now examine the reflectance from a Si-cylinder metasurface with $a=4 r=300 \mathrm{~nm}$ in the optical domain around the spectral region where there is no diffractive order other than the zero-order, specular one; this is calculated from iCEMD, Eqs. (1), and shown in Fig. 3 as a function of both wavelength $\lambda$ and angle of incidence $\theta$.

Broad bands of zero-reflection (total transmission) are evident that have been associated with Kerker-induced Brewster's (KiBs) effects similar to those discussed in Ref. 29. In addition, broad bands of large specular reflection (recall that $\mathrm{Si}$ is nearly transparent) also appear that are connected to $\mathrm{ED}$ and $\mathrm{MD}$ cylinder resonant modes. 


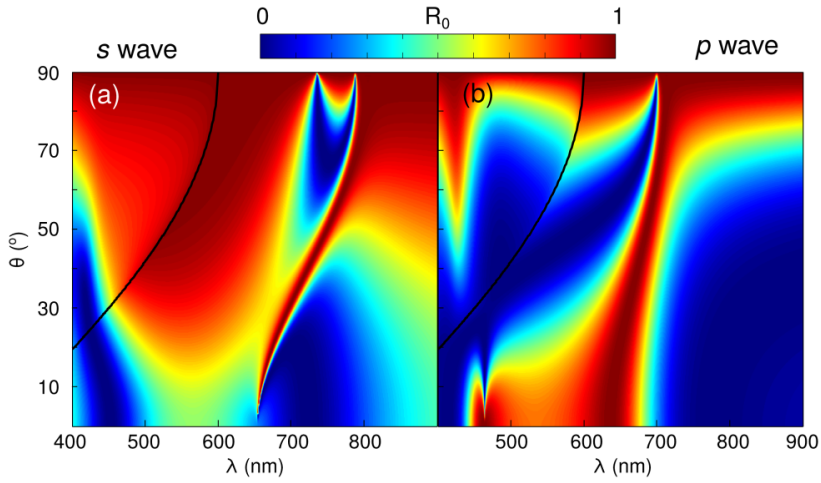

FIG. 3. Contour maps of the specular reflectance calculated through iCEMD for a Si-cylinder metasurface with $r=75 \mathrm{~nm}, a=300 \mathrm{~nm}$, and relative permittivity $\epsilon=12.25$, as a function of the incident wavelength $\lambda$ and angle of incidence $\theta$ for (a) $s$ and (b) $p$ polarization. The diffractive region lies above the black solid curves in the upper left corners.

Nonetheless, we focus here on the emergence of very narrow resonances (especially those close to normal incidence), evident from Fig. 3, that appear despite the fact that they also stem from those broad (Mie) resonances of the isolated cylinders (cf. Fig. 2).

Let us study these narrow resonant modes in detail, by plotting spectral reflectances for fixed angles of incidence in Fig. 4. We chose near normal angles of incidence for both polarizations. All zero-reflection bands are marked, indicating their origin: broad transmission (KiBs) bands described by lattice Kerker conditions, ${ }^{29}$

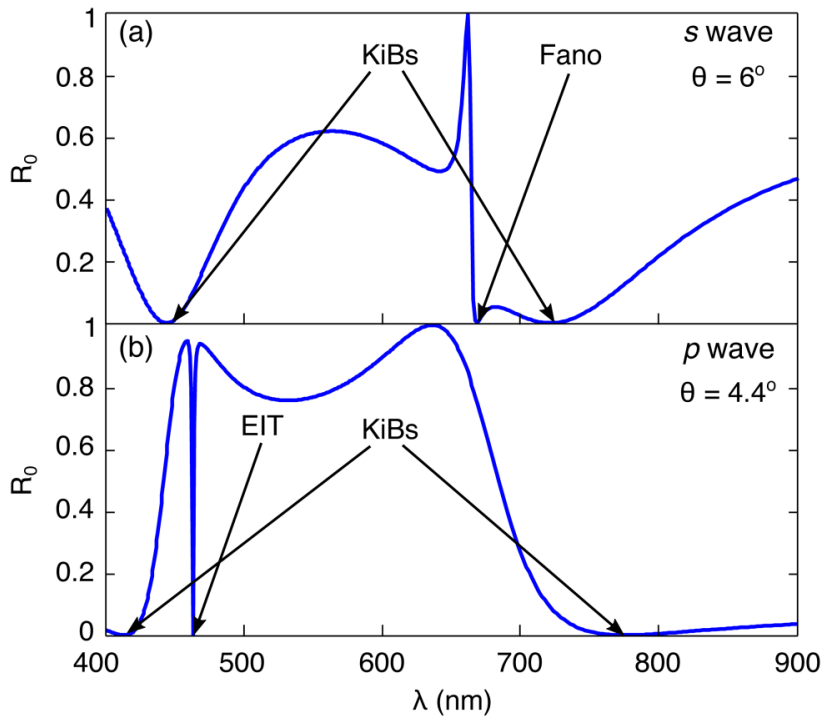

FIG. 4. Specular reflectance spectra from Fig. 3 for fixed angles of incidence. (a) $s$ waves at $\theta=6^{\circ}$; (b) $p$ waves at $\theta=4.4^{\circ}$. Kerker-induced Brewster's (zero reflectance) bands are marked as KiBs; narrow Fano resonance at $\lambda \sim 655 \mathrm{~nm}$ and EIT dip at $\lambda \sim 464 \mathrm{~nm}$ are also highlighted. in contrast to narrow Fano/electromagnetically-induced transparency (EIT) resonances. Such resonances are very narrow, and for $s$ waves are extremely asymmetric, thus exhibiting Fano line shapes similar to those discussed in connection with dielectric nanostructures and metasurfaces. ${ }^{3,9,36,51}$ To this end, we make use of the generalized Fano formula, ${ }^{52}$

$$
\begin{gathered}
f(\varepsilon)=\sigma(\varepsilon) \frac{(\varepsilon+q)^{2}}{\varepsilon^{2}+1}, \\
\varepsilon=\frac{\omega-\omega_{0}}{\frac{1}{2} \Gamma}, \\
\sigma(\varepsilon)=\left(\frac{k^{2}}{2 k a \cos \theta}\right)^{2}\left|\widetilde{\alpha}_{x}-\widetilde{\alpha}_{y} \cos ^{2} \theta\right|^{2},
\end{gathered}
$$

and zoom in them in the frequency domain in Fig. 5. Specifically, the clearly asymmetric line shape for $s$ waves appearing in Fig. 5(a) at $\lambda \sim 659 \mathrm{~nm}\left(\omega_{0}=1.89 \mathrm{eV}\right)$ yields a Fano parameter of $q=1.74$ and a quality factor of $Q \equiv \frac{\omega_{0}}{\Gamma}=420$. On the other hand, the line shape for $p$ waves appearing in Fig. 5(b) at $\lambda \sim 464 \mathrm{~nm}$ $\left(\omega_{0}=2.67 \mathrm{eV}\right)$ resembles a narrow electromagnetically-induced transparency (EIT) window, which can in turn be viewed as a Fano resonance for vanishing $q$ parameter; ${ }^{3}$ indeed, it yields $q=0.01$ with a quality factor of $Q=382$.

Furthermore, upon inspecting the analytical expression of the reflectance, Eqs. (1) and (2), it can be seen that the narrow Fano resonance for $s$ waves stems mainly from: first, a narrow resonant mode arising from the hybridization (accounted for the lattice factor $\gamma^{(s)}$ ) of the transversal MD mode at $\lambda \sim 700 \mathrm{~nm}$ (see Fig. 2), leading to a narrow surface lattice resonance; second, the latter becomes strongly asymmetric upon interfering with a much broader mode, which includes contributions from both the longitudinal ED and the above mentioned transversal MD mode [thus to both $\widetilde{\alpha}_{x}, \widetilde{\alpha}_{z}$ in Eq. (1)]. The interpretation is further confirmed by the dipole moments induced on the cylinders shown in the inset of Fig. 5(a). The electric dipole moment $p_{x}$ (longitudinal ED mode) at normal incidence is a smooth continuous function (blue dashed curve). However, at non-normal incidence, $p_{x}$ hybridized with $m_{z}$ (transversal MD mode) through the coupling term $\gamma^{(s)}$, leading to a Fano resonance also in the electric dipole moment (blue solid curve). The other transversal MD mode $\left(m_{y}\right)$ acts as an additional background but does not play a relevant role in the asymmetry of the profile, i.e., without the longitudinal ED mode the transmittance would be more symmetric as in Fig. 5(b).

The near field at different frequencies is also represented in the bottom part of Fig. 5(a). Since at resonance the near field is enhanced, being more than an order of magnitude higher than the incoming plane wave, the field is plotted in the logarithm scale. At $R_{0}=1$, it is shown that there is no transmitted wave, creating an interference in the backward direction between the incident wave and the reflected wave. In contrast, at $R_{0}=0$, the field is enhanced close to the particles, but they do not radiate to the far field so that the incoming plane wave is transmitted. In both cases, though, the near-field pattern resembles that of a MD oriented along the $\mathrm{z}$ axis. Thus, the transversal MD $m_{z}$ mode is responsible for the high-Q 
(a)
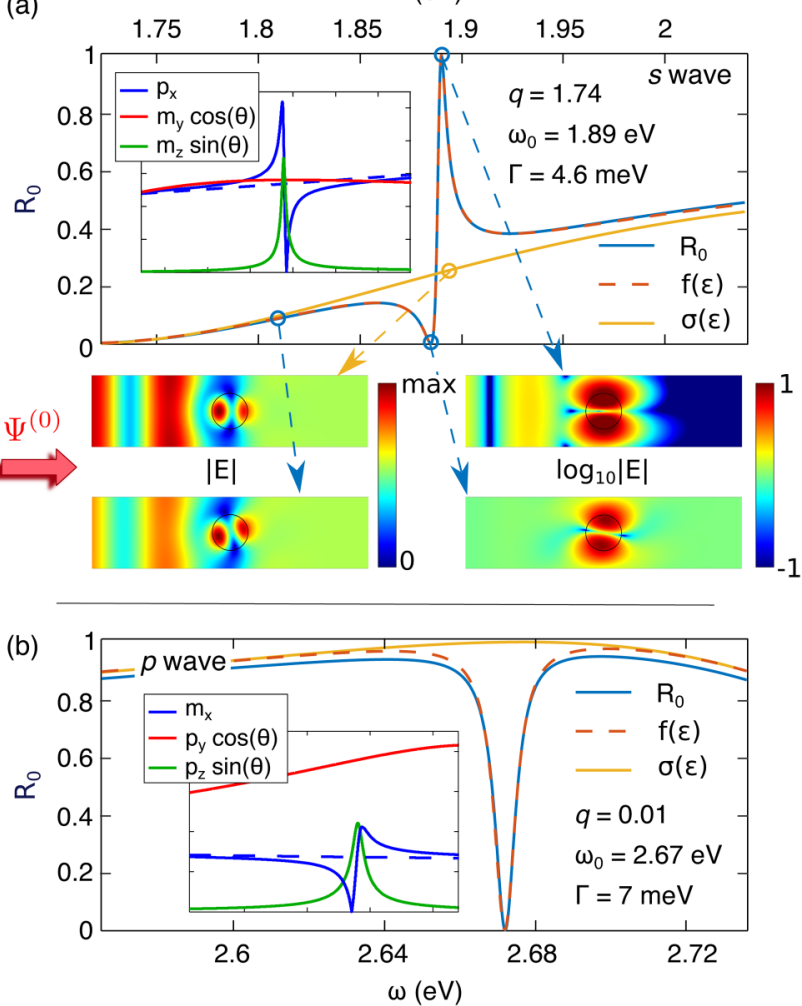

FIG. 5. Same as Fig. 4 but zooming in the spectral regions of Fano and EIT resonances: (a) $s$ waves at $\theta=4.4^{\circ}$; (b) $p$ waves at $\theta=6^{\circ}$. Fits of the spectra to the Fano formula (2) are also shown, with corresponding fitting parameters. The insets represent the absolute value of the dipolar moments induced on the cylinders multiplied by the value of their far field radiation patterns at the angle $\theta$. In addition, near-field plots at different frequencies are shown for $s$ waves: in the latter, the incident plane wave propagates from left to right.

resonance. In addition, the near field at normal incidence is shown in normal linear scale, together with the off-resonance field at $\theta=6^{\circ}$. Both near-field patterns present similar features, given by the interference between the $p_{x}$ and $m_{y}$ dipole.

For $p$ polarization, however, the contribution from the longitudinal (MD) is negligible, $\widetilde{\alpha}_{x}<\widetilde{\alpha}_{y}, \widetilde{\alpha}_{z}$ in Eq. (2c). EIT stems entirely from the transversal ED modes at $\lambda \sim 460 \mathrm{~nm}$ (see Fig. 2). Lattice coupling of such transversal ED modes results then in both a broad and a narrow surface lattice mode $\left[\widetilde{\alpha}_{y}\right.$ and $\widetilde{\alpha}_{z}$, respectively, in Eq. (1)], the interference of which (antiphase) leads to EIT. From the induced dipole moment shown in the inset of Fig. 5(b), it is clear that there is also a hybridization of $m_{x}$ with $p_{z}$ through $\gamma^{(p)}$, but since $\widetilde{\alpha}_{x}$ is small, it is not manifested in the far field.

It should be mentioned that both narrow modes vanish at normal incidence with vanishing width (see Fig. 3 near $\theta \sim 0^{\circ}$ ). This is a clear evidence that they become bound states in the continuum $^{34-36,53,54}$ with diverging Q-factor that will be investigated elsewhere.
On the other hand, as the angle of incidence increases (see Fig. 3), those modes behave differently. For $s$ waves. the Fano mode shifts to larger wavelengths due to the lattice and separates from the broad resonance of the longitudinal mode, becoming slightly broader. For even bigger angles of incidence, $\theta>45^{\circ}$, the roles of the transversal modes are swapped, i.e., $\widetilde{\alpha}_{y}$ becomes narrower than $\widetilde{\alpha}_{z}$, leading to a sign flip of the Fano factor. For $p$ waves, the EIT mode becomes broader and the broad transversal ED mode diminishes (again, $\widetilde{\alpha}_{y}$ and $\widetilde{\alpha}_{z}$ swap their role). Close to grazing angles, the narrow transversal ED mode (now $\widetilde{\alpha}_{y}$ ) shifts toward longer wavelengths where $\widetilde{\alpha}_{x}$ is not negligible. Hence, the lattice couples such longitudinal MD mode with the transversal ED mode (given by $\left.\gamma^{(p)}\right)$ leading to an asymmetric interference.

\section{REFRACTIVE-INDEX SENSING}

In light of the asymmetry and narrowness of the above Fano resonances, we now investigate their dependence on the refractive index of the surrounding medium $n_{e}$. We thus calculate through our iCEMD, the reflectance of a $\mathrm{Si}$ nanocylinder metasurface ( $r=75 \mathrm{~nm}, a=300 \mathrm{~nm}$, and relative permittivity $\epsilon=12.25)$, in the spectral region close to the $s$-polarization Fano resonance shown in Fig. 5(a). This is done in Fig. 6(a) for a value of $n_{e}$ close to that of water, since refractive-index sensors are often used in aqueous solutions. A red-shift of the Fano resonance is observed as compared to Fig. 5(a) due to the higher refractive index of the surrounding medium, as expected. Indeed, this also red-shifts and smears out the broad resonance at shorter wavelengths; thus, the narrow (transverse MD) surface lattice resonance originating the Fano resonance becomes less asymmetric due to the absence of broad background to
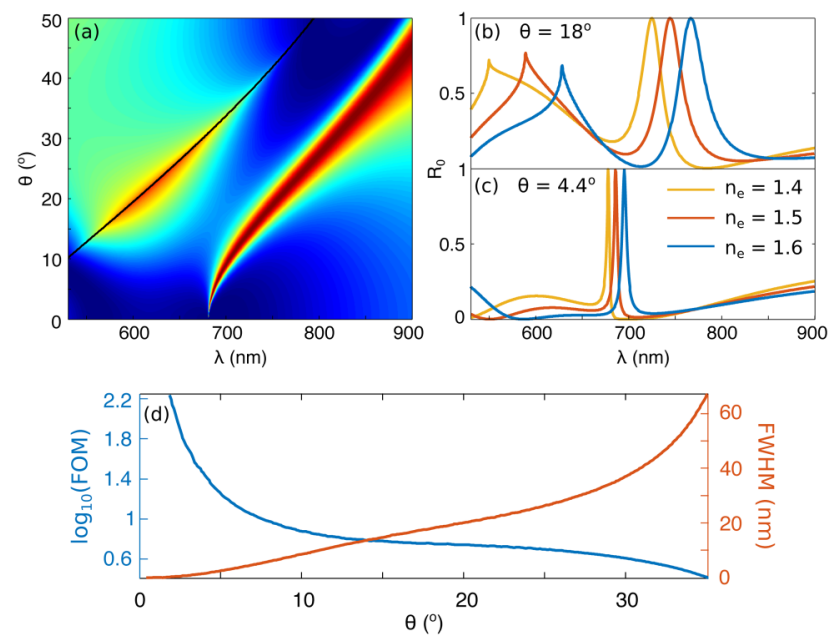

FIG. 6. (a) Specular reflectance as in Fig. 3, but assuming a surrounding medium with $n_{e}=1.5$ instead of vacuum, and only for $s$ polarization and a narrower spectral/angular regime. (b) and (c) Specular reflectance spectra at fixed angles of incidence $\theta=4.4^{\circ}, 18^{\circ}$, as in Fig. 4(a), but for three different values of the refractive index of the surrounding medium: $n_{e}=1.4,1.5,1.6$. (d) FWHM and figure of merit as a function of the angle of incidence for the Fano resonance in the s-polarized reflectance spectra. 
interfere with. Incidentally, such reflectance map with a single narrow resonance reveals a clear bound state in the continuum with diverging Q-factor at $\lambda=680 \mathrm{~nm}$ upon approaching normal incidence, very similar to that described in Ref. 36.

Even though diverging Q-factors are ideal for sensing, we focus on angles of incidence at which the resonance, while being narrow, still yields an observable reflectance signal; recall also that specular reflectance poses experimental difficulties (not so in transmission). This is shown in Figs. 6(b) and 6(c) for two angles of incidence $\theta=4.4^{\circ}, 18^{\circ}$, including three different values of the refractive index of the surrounding medium to observe the sensing performance. Although the shift with varying refractive index is not very large, recall that the relevant magnitude in refractive-index sensing is, however, the figure of merit, f.o.m, defined as the ratio between the sensitivity $S_{\lambda}$ and the full-width at half-maximum FWHM, namely,

$$
\text { f.o.m }=S_{\lambda} / \text { FWHM }=(\Delta \lambda / \text { RIU }) / F W H M,
$$

where RIU stands for refractive-index unit. For the sake of clarity, we explicitly plot in Fig. 6(d) both the FWHM and the resulting figures of merit for varying angle of incidence. Note that the lattice resonance shift induced by the change of the embedding refractive index is substantially larger than that expected for the resonances of isolated HRI nanoparticles. ${ }^{55,56}$ The extremely narrow Fano line shapes thus clearly boost f.o.m. $\sim 200$, making these simple Si-based configurations suitable candidates for refractive-index sensing.

\section{CONCLUDING REMARKS}

To summarize, we have analyzed the optical response of a metasurface consisting of an array of Si nanocylinders of interest in nanophotonics for cylinder sizes and spectral domains where the cylinder response can be described by their electric and magnetic polarizabilities. Thus, we exploit a previously developed coupled electric/magnetic dipole theory for infinite planar arrays. ${ }^{29}$ Special interest has been paid to the emergence of Fano resonances (and EIT) in the reflectance spectra, closely related to bound states in the continuum. Indeed, we demonstrate analytically that strong interference between modes is evidenced though a varied phenomenology, including extremely narrow EIT-like and Fano resonances with Q-factors exceeding $10^{3}$. By looking into the dependence of those narrow asymmetric resonances on the surrounding medium, we explore the performance of those Si nanocylinder metasurfaces as refractive-index sensors; figures of merit f.o.m. $\geq 10^{2}$ are predicted. In this manner, we have analytically described the appearance of high-Q Fano resonances in such simple-geometry, Si-based metasurfaces, showing that they hold potential, in turn, for refractive-index sensing devices at the nanoscale; e.g., a simple practical device, easy to fabricate, could be a $\mathrm{Si}$ nanobeam array deposited on a silica substrate.

\section{ACKNOWLEDGMENTS}

Financial support from the Spanish Ministerio de Economía, Industria y Competitividad through the grants LENSBEAM (Nos. FIS2015-69295-C3-2-P and FIS2015-69295-C3-3-P), NANOTOPO
(No. FIS2017-91413-EXP), and FPU PhD Fellowship (No. FPU15/ 03566) is acknowledged.

\section{REFERENCES}

${ }^{1}$ A. E. Miroshnichenko, S. Flach, and Y. S. Kivshar, Rev. Mod. Phys. 82, 2257 (2010).

${ }^{2}$ B. Luk'yanchuk, N. I. Zheludev, S. A. Maier, N. J. Halas, P. Nordlander, H. Giessen, and C. T. Chong, Nat. Mater. 9, 707 (2010).

${ }^{3}$ M. F. Limonov, M. V. Rybin, A. N. Poddubny, and Y. S. Kivshar, Nat. Photonics 11, 543 (2017).

${ }^{4}$ V. Giannini, A. I. Fernández-Domínguez, S. C. Heck, and S. A. Maier, Chem. Rev. 111, 3888 (2011).

${ }^{5}$ M. Rahmani, B. Luk'yanchuk, and M. Hong, Laser Photon. Rev. 7, 329 (2013).

${ }^{6}$ F. López-Tejeira, R. Paniagua-Domínguez, R. Rodríguez-Oliveros, and J. A. Sánchez-Gil, New J. Phys. 14, 023035 (2012).

${ }^{7}$ P. Fan, Z. Yu, S. Fan, and M. L. Brongersma, Nat. Mater. 13, 471 (2014).

${ }^{8}$ N. Verellen, F. López-Tejeira, R. Paniagua-Domínguez, D. Vercruysse, D. Denkova, L. Lagae, P. Van Dorpe, V. V. Moshchalkov, and J. A. Sánchez-Gil, Nano Lett. 14, 2322 (2014).

${ }^{9}$ D. R. Abujetas, M. A. G. Mandujano, E. R. Méndez, and J. A. Sánchez-Gil, ACS Photonics 4, 1814 (2017).

${ }^{10} \mathrm{C}$. Wu, A. B. Khanikaev, R. Adato, N. Arju, A. A. Yanik, H. Altug, and G. Shvets, Nat. Mater. 11, 69 (2011).

${ }^{11}$ R. Singh, W. Cao, I. Al-Naib, L. Cong, W. Withayachumnankul, and W. Zhang, Appl. Phys. Lett. 105, 171101 (2014).

${ }^{12}$ Z.-J. Yang, T. J. Antosiewicz, R. Verre, F. J. García de Abajo, S. P. Apell, and M. Käll, Nano Lett. 15, 7633 (2015).

${ }^{13}$ S. Yuan, X. Qiu, C. Cui, L. Zhu, Y. Wang, Y. Li, J. Song, Q. Huang, and J. Xia, ACS Nano 11, 10704 (2017).

${ }^{14}$ A. B. Evlyukhin, C. Reinhardt, A. Seidel, B. S. Luk'yanchuk, and B. N. Chichkov, Phys. Rev. B 82, 45404 (2010).

${ }^{15}$ A. García-Etxarri, R. Gómez-Medina, L. S. Froufe-Pérez, C. López, L. Chantada, F. Scheffold, J. Aizpurua, M. Nieto-Vesperinas, and J. J. Sáenz, Opt. Express 19, 4815 (2011).

${ }^{16}$ A. I. Kuznetsov, A. E. Miroshnichenko, M. L. Brongersma, Y. S. Kivshar, and B. Luk'yanchuk, Science 354, aag2472 (2016).

${ }^{17}$ Y. Kivshar and A. Miroshnichenko, Opt. Photonics News 28, 24 (2017).

${ }^{18}$ R. Paniagua-Domínguez, F. López-Tejeira, R. Marqués, and J. A. Sánchez-Gil, New J. Phys. 13, 123017 (2011).

${ }^{19}$ R. Paniagua-Domínguez, L. S. Froufe-Pérez, J. J. Sáenz, and J. A. Sánchez-Gil, Phys. Rev. B 91, 235120 (2015).

${ }^{20}$ R. Paniagua-Domínguez, D. R. Abujetas, and J. A. Sánchez-Gil, Sci. Rep. 3, 1507 (2013).

${ }^{21}$ P. Moitra, Y. Yang, Z. Anderson, I. I. Kravchenko, D. P. Briggs, and J. Valentine, Nat. Photonics 7, 791 (2013).

${ }^{22}$ Y. Yang, W. Wang, P. Moitra, I. I. Kravchenko, D. P. Briggs, and J. Valentine, Nano Lett. 14, 1394 (2014).

${ }^{23}$ P. Moitra, B. a. Slovick, Z. Gang Yu, S. Krishnamurthy, and J. Valentine, Appl. Phys. Lett. 104, 171102 (2014).

${ }^{24}$ P. Moitra, B. A. Slovick, W. Li, I. I. Kravchencko, D. P. Briggs, S. Krishnamurthy, and J. Valentine, ACS Photonics 2, 692 (2015).

${ }^{25}$ S. J. Kim, J. Park, M. Esfandyarpour, E. F. Pecora, P. G. Kik, and M. L. Brongersma, Nano Lett. 16, 3801 (2016).

${ }^{26} \mathrm{~L}$. Li, J. Wang, H. Ma, J. Wang, M. Feng, H. Du, M. Yan, J. Zhang, S. Qu, and Z. Xu, Appl. Phys. Lett. 108, 122902 (2016).

${ }^{27}$ R. Paniagua-Domínguez, Y. F. Yu, A. E. Miroshnichenko, L. A. Krivitsky, Y. H. Fu, V. Valuckas, L. Gonzaga, Y. T. Toh, A. Y. S. Kay, B. Luk'yanchuk, and A. I. Kuznetsov, Nat. Commun. 7, 10362 (2016).

${ }^{28}$ S. Liu, A. Vaskin, S. Addamane, B. Leung, M.-C. Tsai, Y. Yang, P. P. Vabishchevich, G. A. Keeler, G. Wang, X. He, Y. Kim, N. F. Hartmann, H. Htoon, S. K. Doorn, M. Zilk, T. Pertsch, G. Balakrishnan, M. B. Sinclair, I. Staude, and I. Brener, Nano Lett. 18, 6906 (2018). 
${ }^{29}$ D. R. Abujetas, J. A. Sánchez-gil, and J. J. Sáenz, Opt. Express 26, 31523 (2018).

${ }^{30}$ Z. Zhou, J. Li, R. Su, B. Yao, H. Fang, K. Li, L. Zhou, J. Liu, D. Stellinga, C. P. Reardon, T. F. Krauss, and X. Wang, ACS Photonics 4, 544 (2017).

${ }^{31}$ F. Aieta, M. A. Kats, P. Genevet, and F. Capasso, Science 347, 1342 (2015).

${ }^{32}$ M. Khorasaninejad, W. T. Chen, R. C. Devlin, J. Oh, A. Y. Zhu, and F. Capasso, Science 352, 1190 (2016).

${ }^{33}$ Y. Yang, I. I. Kravchenko, D. P. Briggs, and J. Valentine, Nat. Commun. 5, 5753 (2014).

${ }^{34}$ D. C. Marinica, A. G. Borisov, and S. V. Shabanov, Phys. Rev. Lett. 100, 183902 (2008).

${ }^{35}$ E. N. Bulgakov and A. F. Sadreev, Phys. Rev. A 90, 53801 (2014).

${ }^{36}$ D. R. Abujetas, A. Barreda, F. Moreno, J. J. Saenz, A. Litman, J.-M. Geffrin, and J. A. Sanchez-Gil, e-print arxiv:1902.07148 (2019).

${ }^{37}$ B. García-Cámara, R. Gómez-Medina, J. J. Sáenz, and B. Sepúlveda, Opt. Express 21, 23007 (2013).

${ }^{38}$ Y. Wang, B. Gao, K. Zhang, K. Yuan, Y. Wan, Z. Xie, X. Xu, H. Zhang, Q. Song, L. Yao, X. Fang, Y. Li, W. Xu, J. Zhang, and L. Dai, ACS Photonics 4, 688 (2017).

${ }^{39}$ D. Visser, B. D. Choudhury, I. Krasovska, and S. Anand, Opt. Express 25, 12171 (2017)

${ }^{40}$ K. A. Willets and R. P. Van Duyne, Annu. Rev. Phys. Chem. 58, 267 (2007).

${ }^{41}$ K. M. Mayer and J. H. Hafner, Chem. Rev. 111, 3828 (2011).

42. M. Pryce, Y. A. Kelaita, K. Aydin, and H. A. Atwater, ACS Nano 5, 8167 (2011).
${ }^{43}$ F. López-Tejeira, R. Paniagua-Domínguez, and J. A. Sánchez-Gil, ACS Nano 6, 8989 (2012).

${ }^{44}$ E. Baquedano, M. U. González, R. Paniagua-Domínguez, J. A. Sánchez-Gil, and P. A. Postigo, Opt. Express 25, 15967 (2017).

${ }^{45}$ A. Tittl, A. Leitis, M. Liu, F. Yesilkoy, D.-Y. Choi, D. N. Neshev, Y. S. Kivshar, and H. Altug, Science 360, 1105 (2018).

${ }^{46}$ Y. Yang, A. E. Miroshnichenko, S. V. Kostinski, M. Odit, P. Kapitanova, M. Qiu, and Y. S. Kivshar, Phys. Rev. B 95, 165426 (2017).

${ }^{47}$ M. Laroche, S. Albaladejo, R. Gómez-Medina, and J. J. Sáenz, Phys. Rev. B 74, 245422 (2006).

${ }^{48}$ Y. Chen, Y. Zhang, and A. Femius Koenderink, Opt. Express 25, 21358 (2017).

${ }^{49}$ G. Brönstrup, N. Jahr, C. Leiterer, A. Csáki, W. Fritzsche, and S. Christiansen, ACS Nano 4, 7113 (2010).

${ }^{50}$ D. Sell, J. Yang, S. Doshay, K. Zhang, and J. A. Fan, ACS Photonics 3, 1919 (2016).

${ }^{51}$ M. V. Rybin, D. S. Filonov, P. A. Belov, Y. S. Kivshar, and M. F. Limonov, Sci. Rep. 5, 8774 (2015).

${ }^{52}$ T. K. Fang and T. N. Chang, Phys. Rev. A 57, 4407 (1998).

${ }^{53}$ K. Koshelev, S. Lepeshov, M. Liu, A. Bogdanov, and Y. Kivshar, Phys. Rev. Lett. 121, 193903 (2018).

${ }^{54}$ D. R. Abujetas, N. van Hoof, S. ter Huurne, J. G. Rivas, and J. A. Sánchez-Gil, e-print arxiv:1901.03122 (2019).

${ }^{55}$ A. Polman and J. van de Groep, Opt. Express 21, 26285 (2013).

${ }^{56}$ Z.-J. Yang, Q. Zhao, and H. Jun, J. Appl. Phys. 125, 063103 (2019). 